Original Article

\title{
Influence of transcutaneous electrical nerve stimulation on weight distribution in lower leg muscles
}

\author{
Hyeonsook Rhee, PhD, $\mathrm{OT}^{1)}$, Jaeho Yu, PhD, PT ${ }^{2)}$, Kinun Cho, PhD, $\mathrm{PT}^{3)^{*}}$ \\ 1) Department of Occupational Therapy, Shinsung University, Republic of Korea \\ 2) Department of Physical Therapy, Sun Moon University, Republic of Korea \\ 3) Department of Rehabilitative and Assistive Technology, Korea National Rehabilitation Research \\ Institute, Korea National Rehabilitation Center: 58 Samgaksan-ro, Gangbuk-gu, Seoul 142-070, \\ Republic of Korea
}

\begin{abstract}
Purpose] The purpose of this study was to compare the effects of transcutaneous electrical nerve stimulation (TENS), with and without visual input, on weight distribution following exercise-induced fatigue in the dorsiflexor and plantar flexor muscles of the ankle. [Subjects and Methods] This study had a cross-sectional design. Nineteen healthy adults ( 10 males, 9 females; mean age $21 \pm 0.8$ years) were recruited to participate in a single group repeated measurements study lasting three days. On the first day, following exercise-induced fatigue, the standing position was maintained for 30 minutes, after which the postural sway was measured with eyes open (EO) and eyes closed (EC). On the second day, TENS was applied to the ankle dorsiflexors in the standing position for 30 minutes following exercise-induced fatigue. On the last day, TENS was applied to the plantar flexors, and the postural sway was measured with EO and EC following the same exercise-induced fatigue. [Results] On level terrain, with and without visual input, there was a significant difference between the baseline values and those following TENS on the tibialis anterior. On uneven terrain (simulated by a cushion), with and without visual input, there was a significant difference between the baseline values and those following TENS on the gastrocnemius. [Conclusion] Clinically, during walking on a flat surface for only a short period of time, TENS should be applied to the tibialis anterior. If walking training is performed on a variety of terrains for a longer time, TENS should be applied to the gastrocnemius.
\end{abstract}

Key words: Transcutaneous electrical nerve stimulation (TENS), Weight distribution index (WDI), Visual input

(This article was submitted Dec. 1, 2014, and was accepted Jan. 11, 2015)

\section{INTRODUCTION}

Human postural movement is controlled by multi-sensory inputs from visual, vestibular, and somatic sensory systems. In the standing position, healthy adults reduce the range of motion of their center of mass (COM) by reflexively contracting the tibialis anterior, soleus, and gastrocnemius muscles. This contraction reduces movement at the ankles, resulting in a lower amplitude and higher frequency postural sway ${ }^{1)}$. These postural controls vary linearly with height and are more pronounced in the anteroposterior than mediolateral directions ${ }^{2}$. They are associated with enhanced soleus tendon reflexes but not with changes in cortical sensory or motor evoked potentials. When standing at heights, people also experience autonomic activation, greater fear of falling,

\section{*Corresponding author. Kihun Cho (E-mail: mamiya34@} gmail.com)

C 2015 The Society of Physical Therapy Science. Published by IPEC Inc. This is an open-access article distributed under the terms of the Creative Commons Attribution Non-Commercial No Derivatives (by-ncnd) License $<$ http://creativecommons.org/licenses/by-nc-nd/3.0/>. and altered cognitive appraisals about their postural control.

Among the sensory inputs for postural control, visual acuity is reported to reduce the postural sway by limiting the external elements for a fall ${ }^{3)}$. The plantar flexors and dorsiflexors interact with each other in opposition as agonists and antagonists to reduce the postural sway and are effective in stabilizing posture ${ }^{4}$. Additionally, changes in somatic sensory input due to muscle fatigue are reported to cause deficits of neuromuscular and postural control ${ }^{5}$. Prior studies have explained the relationship between fatigue and altered neuromuscular control; muscles affected by fatigue send input signals with a reduced transmission rate so that decreased output signals limit the formation of effective actions ${ }^{6}$. Application of transcutaneous electrical neuromuscular stimulation (TENS) improves postural instability and ameliorates muscle power reduction caused by muscle fatigue $^{7}$.

Many previous studies have analyzed the dorsiflexors and plantar flexors as muscles with tremendously important roles in walking, with agonist and antagonist functions, but differences caused by the presence or absence of visual input are inadequately evaluated. Prior studies have applied TENS mostly to the thigh muscles, and almost no studies 
have compared TENS applications to other lower extremity muscles. Therefore, this study aims to ascertain differences in postural sway, according to the presence or absence of visual input, with the application of TENS to the dorsiflexor and plantar flexor muscles.

\section{SUBJECTS AND METHODS}

A cross-sectional, single-blinded, one group repeated measures study was performed. The project was approved by the University of Sun Moon Research Ethics Review Committee (SM-201410-029-2, 24 November 2014), and the study protocol was conducted in strict accordance with the Declaration of Helsinki. Written informed consent was obtained from each participant. Study participants were selected from healthy adult men and women who agreed to participate after an advance survey. There were a total of 19 participants including 10 males and 9 females (mean age $21 \pm 0.8$ years). The mean height was $167 \pm 9.70 \mathrm{~cm}$, and mean weight was $60.40 \pm 11.70 \mathrm{~kg}$. In the study, measurements were performed in one group for a total of three days. On the first day, to cause leg fatigue, participants were asked to flex and extend the foot 40 times, and the postural sway was measured using TETRAX (Sunlight Co. Ltd, Israel) ${ }^{8)}$. Measurements were performed with eyes open (EO) for 32 seconds, and later, the measurements were repeated with eyes closed (EC). After the experiment on the first day, the participants rested for the remainder of the day to avoid any influence on subsequent experiments. On the second day, after performing fatigue-inducing movements as mentioned above, TENS (STT-500, Stratek, Korea) was applied with a frequency of $100 \mathrm{~Hz}$ and current of $8.4 \mathrm{~mA}$ to the tibialis anterior for 30 minutes during standing. After the application of TENS, the postural control was measured using TETRAX. On the third and final day of the study, after performing fatigue-causing movements during the same time period, TENS was applied with the same frequency and intensity to the gastrocnemius, and the postural sway was measured. Following this, the weight distribution index (WDI) value was obtained. All measurement values were statistically processed using SPSS 18.0 for Windows. Measurements were obtained at baseline and following TENS application to the dorsiflexor and plantar flexor muscles in the standing posture, for with and without visual input conditions, and differences between the measurements were compared. One-way repeated ANOVA was used for analysis, and the
Bonferroni method was employed as a post-hoc test for differences between the variables. The statistical significance level for all analyses was set as a $p$ value $<0.05$.

\section{RESULTS}

Table 1 shows the change in WDI after each application of TENS following exercise-induced fatigue. On level ground, with and without visual input, there was a significant difference between baseline values and those following TENS on the tibialis anterior $(\mathrm{p}<0.05)$. In the pillow condition (simulating uneven ground), with and without visual input, there was a significant difference between baseline values and those following TENS on the gastrocnemius $(p<0.05)$.

\section{DISCUSSION}

Many previous studies have examined the role of the tibialis anterior as a dorsiflexor. Ebig et al. studied plantar flexor fatigue following movements of the peroneal and tibialis anterior muscles in healthy adult subjects and reported no significant differences in the postural sway after exercise ${ }^{9)}$. Additionally, according to Tilp et al., changes in the aponeurosis length between concentric and eccentric contraction of the tibialis anterior showed no significant difference between groups $^{10)}$. The tibialis anterior is one of most important muscles in postural control during standing, and these results are thought to represent the normal condition, regardless of the eyes open or closed state. The tibialis anterior may be adequate to maintain postural control on its own on level ground as gastrocnemius activity is significantly less.

However, the gastrocnemius is more active than the tibialis anterior on unstable ground as indicated by the pillow condition in our study result. There are several reasons for the TENS application on the gastrocnemius to be more effective in reducing the postural sway. First, there are more motor nerve cells and movement units present as the gastrocnemius muscle is larger ${ }^{11)}$. Second, the main supporting muscles when a person stands for a long time are reported to be the plantar flexors ${ }^{12)}$. In addition, the plantar flexors include the soleus, and the gastrocnemius is reported to control movement by becoming active first when the erect standing posture is maintained ${ }^{4}$. Therefore, if the patient is on a flat surface for a short period of time, it will be necessary to apply TENS to the tibialis anterior. If walking training is performed on a variable or uneven terrain for a long period of

Table 1. Changes in the weight distribution index after each application of transcutaneous electrical stimulation following exercise-induced fatigue

\begin{tabular}{llccc}
\hline & & Baseline & Tibialis anterior & Gastrocnemius \\
\hline \multirow{2}{*}{ Normal } & Eyes open & $5.9 \pm 2.4$ & $4.2 \pm 1.7^{*}$ & $10.0 \pm 1.7$ \\
& Eyes closed & $5.8 \pm 2.2$ & $4.2 \pm 2.0^{*}$ & $4.6 \pm 1.7$ \\
\multirow{2}{*}{ Pillow } & Eyes open & $5.8 \pm 2.7$ & $4.6 \pm 1.5$ & $3.4 \pm 1.7^{\#}$ \\
& Eyes closed & $5.3 \pm 2.1$ & $4.2 \pm 2.0$ & $3.6 \pm 2.0^{\#}$ \\
\hline
\end{tabular}

All variables are presented as mean \pm SD. Tibialis anterior: application of TENS on tibialis anterior, gastrocnemius: application of TENS on gastrocnemius, ${ }^{*}$ indicates a significant difference between baseline and tibialis anterior $(\mathrm{p}<0.05)$. " indicates a significant difference between baseline and gastrocnemius $(p<0.05)$ 
time, TENS should be applied to the gastrocnemius. TENS is expected to be helpful in improving postural control by reducing muscle fatigue. Moreover, improved postural control during walking could help prevent falls. Future studies on various measures to reduce postural sway are required.

\section{ACKNOWLEDGEMENT}

This work was supported by the Sun Moon University Research Grant in 2014.

\section{REFERENCES}

1) Adkin AL, Frank JS, Carpenter MG, et al.: Postural control is scaled to level of postural threat. Gait Posture, 2000, 12: 87-93. [Medline] [CrossRef]

2) Toussaint HM, Commissaris DA, Hoozemans MJ, et al.: Anticipatory postural adjustments before load pickup in a bi-manual whole body lifting task. Med Sci Sports Exerc, 1997, 29: 1208-1215. [Medline] [CrossRef]

3) Vouriot A, Gauchard GC, Chau N, et al.: Sensorial organisation favouring higher visual contribution is a risk factor of falls in an occupational setting. Neurosci Res, 2004, 48: 239-247. [Medline] [CrossRef]

4) Di Giulio I, Maganaris CN, Baltzopoulos V, et al.: The proprioceptive and agonist roles of gastrocnemius, soleus and tibialis anterior muscles in maintaining human upright posture. J Physiol, 2009, 587: 2399-2416. [Medline] [CrossRef]

5) Gimmon Y, Riemer R, Oddsson L, et al.: The effect of plantar flexor muscle fatigue on postural control. J Electromyogr Kinesiol, 2011, 21: 922 928. [Medline] [CrossRef]

6) Bellew JW, Fenter PC: Control of balance differs after knee or ankle fatigue in older women. Arch Phys Med Rehabil, 2006, 87: 1486-1489. [Medline] [CrossRef]

7) Cho HY, Lee SH, In TS, et al.: Effect of transcutaneous electrical nerve stimulation (TENS) on changes in postural balance and muscle contraction following muscle fatigue. J Phys Ther Sci, 2011, 23: 899-903. [CrossRef]

8) Roerdink M, Hlavackova P, Vuillerme N: Effects of plantar-flexor muscle fatigue on the magnitude and regularity of center-of-pressure fluctuations. Exp Brain Res, 2011, 212: 471-476. [Medline] [CrossRef]

9) Ebig M, Lephart SM, Burdett RG, et al.: The effect of sudden inversion stress on EMG activity of the peroneal and tibialis anterior muscles in the chronically unstable ankle. J Orthop Sports Phys Ther, 1997, 26: 73-77. [Medline] [CrossRef]

10) Tilp M, Steib S, Herzog W: Length changes of human tibialis anterior central aponeurosis during passive movements and isometric, concentric, and eccentric contractions. Eur J Appl Physiol, 2012, 112: 1485-1494. [Medline] [CrossRef]

11) Choi YM, Kim BC: Muscle fiber characteristics, myofibrillar protein isoforms, and meat quality. Livest Sci, 2009, 122: 105-118. [CrossRef]

12) Borg F, Finell M, Hakala I, et al.: Analyzing gastrocnemius EMG-activity and sway data from quiet and perturbed standing. J Electromyogr Kinesiol, 2007, 17: 622-634. [Medline] [CrossRef] 